\title{
Acceptability of an mHealth breast cancer risk-reduction intervention promoting risk assessment, education, and discussion of risk in the primary care setting
}

\author{
Celia P. Kaplan ${ }^{1,2}$, Leah Karliner ${ }^{1,2}$, Andrew Lee ${ }^{3}$, Jennifer Livaudais-Toman ${ }^{1}$, Jeffrey A. Tice ${ }^{1}$, \\ Elissa Ozanne ${ }^{4}$
}

${ }^{1}$ Division of General Internal Medicine, University of California, San Francisco, CA, USA; ${ }^{2}$ Multiethnic Health Equity Research Center, University of California, San Francisco, CA, USA; ${ }^{3}$ Department of Radiology and Biomedical Imaging, University of California, San Francisco, CA, USA; ${ }^{4}$ Population Health Science, University of Utah School of Medicine, Salt Lake City, UT, USA

Contributions: (I) Conception and design: CP Kaplan, E Ozanne, L Karliner, JA Tice; (II) Administrative support: CP Kaplan; (III) Provision of study materials or patients: CP Kaplan; (IV) Collection and assembly of data: CP Kaplan, L Karliner, JA Tice; (V) Data analysis and interpretation: J Livaudais-Toman, E Ozanne, CP Kaplan, L Karliner; (VI) Manuscript writing: All authors; (VII) Final approval of manuscript: All authors.

Correspondence to: Celia P. Kaplan, DrPH. Professor, University of California, Gladys Valley Center for Vision, $48016^{\text {th }}$ Street, Box 0856 , San Francisco, CA 94143, USA. Email: celia.kaplan@ucsf.edu.

Background: Breast cancer risk assessment tools and risk reduction strategies have advanced significantly over the past few decades but are underutilized in practice, due in part to limited acceptability by patients and physicians. We implemented a tablet-based Breast Cancer Risk Education Intervention (BreastCARE) tailored towards increasing patients' knowledge about their individual risk of developing breast cancer, increasing patient-physician discussion of breast cancer risk reduction practices, and increasing participation in recommended screening.

Methods: We surveyed patients and physicians who received the BreastCARE intervention and analyzed their satisfaction and acceptability of the intervention. We compared patient satisfaction measures by race/ethnicity and used multivariable logistic regression models to examine the effect of race/ethnicity on measures of patient satisfaction with the tablet-based risk assessment and with the breast cancer risk report. We also compared measures of physician satisfaction by resident vs. attending/NP status. Finally, we identified patients' and physicians' suggestions for implementation.

Results: Overall, both patients and physicians were highly satisfied with BreastCARE, with some variation by patient race/ethnicity and breast cancer risk status. The risk assessment tool and accompanying risk report helped transmit complex information in an efficient way.

Conclusions: Patient self-administered risk assessment with a health education component at the point of care is acceptable for both patients and physicians, and represents a novel approach to facilitating health promotion. This risk assessment tool should be made routine in primary care accompanied by results that are easy for the patient to understand and actionable for the clinician.

Keywords: Physician satisfaction; patient satisfaction; breast cancer risk assessment; implementation; dissemination; communication

Received: 04 March 2020; Accepted: 21 December 2020; Published: 20 October 2021.

doi: $10.21037 /$ mhealth-20-82

View this article at: http://dx.doi.org/10.21037/mhealth-20-82 


\section{Introduction}

Patient self-administered risk assessment with a health education component at the point of care represents a novel approach to facilitate health promotion. Breast cancer risk assessment and risk reduction strategies can easily be adapted to this method. With significant advancements over the past few decades, several validated models and tools now exist to assess predisposition for breast cancer (1-5). Models for breast cancer risk identification of non-hereditary breast cancer include the Gail risk assessment model (6) which assesses breast cancer risk based on a woman's personal medical and reproductive history, age, the history of breast cancer among her first-degree relatives (mother, sisters, daughters), race/ethnicity and prior history of breast biopsy. The Breast Cancer Surveillance Consortium (BCSC) risk assessment model incorporates mammographic breast density into the Gail model $(1,7)$. The breast/ovarian cancer genetics Referral Screening Tool (RST) (8) is an alternative tool that allows for easy screening for hereditary risk in the clinical setting followed by referrals to genetic counseling and testing if necessary.

In conjunction with these risk assessment methods, medical options to reduce risk, including selective estrogen receptor modulators (9-11), aromatase inhibitors (12), and prophylactic surgery (13), are available to women with elevated risk. Despite these advances, tools for assessing risk have been underutilized in the clinical setting (14-16). This may be due in part to limited acceptability by patients and physicians. As a result, many patients do not engage in a discussion of breast cancer risk with their physicians, and many high-risk women are not offered preventive and riskreduction therapies.

Mobile health, also known as mHealth, is the practice of medicine or public health that is supported by mobile devices. Mobile health offers the opportunity to bridge gaps in patient-physician risk discussions by providing women with a tool to assess their breast cancer risk as well as information to educate themselves about risk. In recent years, studies have demonstrated the benefits of integrating mHealth interventions into the healthcare setting and thus, promoting positive patient outcomes (17-21). While few studies have examined the use of breast cancer risk assessment tools in primary care practices (20-23), results suggest there is a great potential to accelerate the collection of risk information using interactive computer programs.

There is a need to understand how such risk communication strategies are accepted among different racial/ethnic groups in order to eliminate potential health disparities, as certain racial/ ethnic groups are at increased risk for breast cancer. The literature has found that Black/African American women are significantly less likely to recognize to be high risk any type of risk reduction therapy compared to White women (14). In addition, Latina and Black/African American women are less likely than White women to have heard of either breast cancer chemoprevention options or genetic counseling (14).

Physicians are instrumental in the dissemination of health information. However, breast cancer risk discussions are quite infrequent across race/ethnic groups, even for women at high risk $(14,15)$. Primary care physicians cite many barriers to the discussion of risk and counseling about risk reduction options, including "not enough time" (14). Barriers among physicians are likely also dependent on the level of experience and familiarity with the topic. However, data are limited to support this assertion and therefore there is a need for further investigation.

To build on the findings suggesting that mHealth has the potential to accelerate collection and discussion of risk information, we implemented Breast Cancer Risk Education Intervention (BreastCARE), a tablet-based breast cancer risk assessment and education intervention for patients and physicians. BreastCARE was found to be effective in increasing patients' knowledge of their individual risk of developing breast cancer, increasing patient-physician discussion of breast cancer risk reduction practices based on individual risk, and increasing participation in recommended screening (24,25). However, effective implementation and dissemination of this type of intervention depends on both patient and physician acceptability and perceptions of usability in busy primary care practices.

In this analysis, we evaluate patient and physician acceptability of the mHealth intervention and examine differences in satisfaction across patient race/ethnicity and physician type.

\section{Methods}

\section{Description of BreastCARE intervention}

The BreastCARE intervention study has been described in detail in a prior publication (24). It was conducted between June 2011 and August 2012 at two general medicine primary care practices, one in an academic medical center and the other in an academic safety-net hospital. Both practices have ethnically and linguistically diverse patient panels and are staffed by faculty and resident physicians. 
The BreastCARE intervention consisted of a tablet-based patient risk assessment tool that generated individually tailored reports for patients and their physicians. The tablet program was designed by Jambeyang Research, an engineering company dedicated to creating health care applications (26).

The project coordinator handed the tablet containing the risk assessment to the patient in the waiting room just prior to a primary care visit. The project coordinator also printed out the tailored patient and physician reports. The tablet program queried patients on breast cancer risk factors in a series of questions written at an eighth-grade reading level. Participants were allowed to skip questions if desired. On average, patients were able to complete the assessment in five minutes while waiting for their visit. Each patient received her individualized report as well as a physician report to hand to her physician at the time of the visit (see https://cdn.amegroups.cn/static/public/mHealth-20-82-1. pdf for examples of the reports, which have not been published previously). The individualized reports, designed with the assistance of a graphic designer, provided education to the patient and physician about breast cancer risk factors and were designed to prompt patient-physician discussion of individual risk for each patient. The report suggested brief preventative recommendations based on the participant's risk factors. For women identified as high risk, the patient report indicated that her risk was "higher than for other women [her] age" and suggested discussing with their physician. Other topics addressed included family history of breast and ovarian cancer, alcohol use, healthy weight, and mammography screening. The physician report provided evidence-based recommendations and suggested appropriate referrals, both based on the patient's risk.

Both the risk assessment and the patient report were available in English, Spanish, traditional Chinese, and simplified Chinese characters to match the patient populations at the two centers. The patient kept the patient report and was able to take it home if she wished to do so.

\section{Procedures}

\section{Patient component}

Patients were eligible to participate if they had an upcoming appointment at one of the participating practice sites during the study period, were between the ages of 40 and 74, spoke English, Spanish, or Chinese (Cantonese or Mandarin), had no personal history of breast cancer, and their physicians did not object to their participation. We also excluded patients if they were unable to complete a telephone survey (e.g., substantial hearing problems). Patients who agreed to participate completed a baseline telephone survey that included basic demographic characteristics (24). At the clinic site, just prior to the primary care visit, women who were randomized to the intervention group took the BreastCARE assessment and received the reports. All data entered in the tablet was saved in the University of California, San Francisco (UCSF), Department of Medicine secure server which follows HIPAA mandates. No data was stored in the tablet to protect patient confidentiality. Within 1 week after the primary care (index) visit, all participants were contacted for a follow-up telephone survey to assess discussion of breast cancer risk and lifestyle behaviors with their physician at the index visit. Intervention participants were also asked about their satisfaction with BreastCARE, including its ease of use and understandability, the topic of this manuscript.

\section{Physician component}

The physician component consisted of a cross-sectional survey of primary care physicians in the two participating practices whose patients were in the intervention arm of the BreastCARE study. Prior to patient recruitment, all practicing physicians were provided with a description of the study and a passive consent form for their participation in the study. After patient recruitment was completed, physicians were sent a study description and invitation to participate in the survey by both mail and email. The mail version contained a paper copy of the survey, while the email had a personalized link to the study website where physicians could complete the survey online. This first contact was followed by reminder emails (again with a personalized link) 2 weeks later. For non-respondents, we sent a second survey mailing and a follow-up reminder via email 1 month later.

The study was conducted in accordance with the Declaration of Helsinki (as revised in 2013). The study was approved by the Institutional Review Board (IRB) of UCSF (project number 10-01288;10-00129) and informed consent was obtained from all participants.

\section{Measures}

\section{Patient component}

Demographic characteristics (baseline survey)

Information collected about patients included self-reported age (less than 50, 50-65), race/ethnicity (Asian, African 
American, Latina, white, American Indian, or other), language of interview (English, Spanish or Chinese), marital status (married/living with a partner $v s$. other), education (high school diploma or less, some college, or college or higher) and access to electronic information including comfort using a computer (very, somewhat, not at all), use of the computer, use of internet and use of email (always, sometimes, never).

Breast cancer risk assessment indicators were elicited via the tablet as part of the intervention delivery. They included age at menarche, age at first birth and menopause, breast biopsy history (yes, no), tamoxifen or raloxifene use (yes, no), Ashkenazi Jewish ancestry (yes, no), and family history of ovarian and breast cancer (list of first-degree family members). For women who had a prior mammogram (yes, no), the breast density from their most recent mammogram report was abstracted from the electronic medical record (EMR).

To estimate objective risk for breast cancer, we used three measures: the RST (Jewish ancestry, history of family breast and ovarian cancer) (8); the Gail Model (6,7), and the BCSC $(1,7)$. Women were considered to be high-risk based on any of the following: (I) a family history threshold for the RST (8), (II) BCSC score in top 5\% of estimated 5-year risk for her age group (1), or (III) Gail score in the top 5\% estimated 5 -year risk for her age group (6). In addition, women between the ages of 40 and 50 were considered high-risk if their Gail or BCSC score was $\geq 1.67$ (9).

\section{Satisfaction}

To evaluate patient satisfaction with the tablet-based assessment tool, respondents were asked to indicate on a four-point Likert scale how easy it was to complete the computer survey (very hard, somewhat hard, somewhat easy, very easy), and how easy the computer survey questions were to understand (very hard, somewhat hard, somewhat easy, very easy). Patients were asked similar questions to evaluate their satisfaction with the risk report: how much they liked it (not at all, not very much, a little, a lot) and how easy it was to understand (very hard, somewhat hard, somewhat easy, very easy).

\section{Preferences}

As noted above, we asked questions regarding computer literacy, including frequency of computer, Internet, and email use. Patients were asked if they preferred to complete the risk assessment on a computer or on paper. We also assessed the preferred location to complete the risk assessment (in the waiting room, at home, either), and the preferred time to receive the breast cancer risk report (before a doctor's visit, after a doctor's visit, either).

\section{Physician component}

\section{Physician and practice characteristics}

We collected the following physician demographic characteristics: gender, self-reported race/ethnicity (Asian American, Black/African American, Latin, White, American Indian, or other), current position [resident, attending physician/nurse practitioner (NP)], and estimated percentage of women patients between the ages of 40 to 74 on their panel. Physician practice characteristics included years practicing medicine and average time (hours) per week spent in direct patient care.

\section{Satisfaction}

We assessed physician satisfaction with both versions (patient and physician) of the BreastCARE risk report, asking whether they recalled receiving the reports and, using a Likert scale (very good, good, fair, poor), their overall rating of each. Physicians were also asked about their perceptions of the reports, specifically, how much they agreed (strongly or somewhat) or disagreed (strongly or somewhat) with positive and negative statements about the reports.

\section{Acceptability of implementation into routine care}

Physicians were asked whether they would want the BreastCARE risk assessment tool and reports (patient and physician) implemented in their practices. They were also asked about the ideal location to deliver breast cancer risk information (primary care or gynecology visit, mammography visit, or at patient's home) and the ideal professional to deliver this information (primary care clinician or gynecologist, trained staff at primary care/ gynecology clinic, radiologist at mammography clinic, trained staff at mammography clinic, or other trained professional by phone).

\section{Statistical analysis}

We used descriptive statistics to profile patient characteristics and measures of patient satisfaction with the intervention. We compared patient satisfaction measures by race/ethnicity using chi-square tests to compare categorical variables and $t$-tests to compare continuous variables across groups. We examined four aspects of patient acceptability including how easy it was to complete the tool (very easy vs. other), how easy it was to understand the questions in the tool (very easy $v s$. other), how much they liked the personalized risk report 
(a lot $v s$. other) and how easy it was to understand the report (very easy $v s$. other).

Multivariable logistic regression models were used to examine the effect of race/ethnicity on measures of patient satisfaction with the tablet-based risk assessment and with the breast cancer risk report, adjusting for clinic site, computer use, age, language, education, and objective breast cancer risk. For the ease of completing the survey, ease of understanding the questions and ease of understanding the report, the outcome indictors were dichotomized as "very easy" versus all other responses. Liking the personalized report was dichotomized as "a lot" versus all other responses.

We also used descriptive statistics to profile physician characteristics and measures of physician satisfaction with the intervention, and compared these satisfaction measures across physicians by resident $v s$. attending/NP status.

A two-sided alpha $=0.05$ was considered the threshold for statistical significance. We analyzed all data using Stata (version 14.2).

\section{Results}

\section{Patient sample}

A total of 603 women were randomized to the intervention group, completed the baseline survey, and took the breast cancer risk assessment. Of these, 573 (95\%) identified as one of the four racial/ethnic groups of interest and completed follow-up surveys. The majority of women were between 50 and 65 years of age $(n=307,54 \%)$, not married or living with a partner $(\mathrm{n}=312,55 \%)$, and had a college degree or higher $(n=223,40 \%)$. Most of the surveys $(n=500$, $87 \%)$ were conducted in English. Nearly 68\% ( $n=383)$ of women reported being somewhat or very comfortable using the computer, and a majority said they sometimes or always used the computer $(n=397,69 \%)$, the internet $(n=389$, $68 \%)$, and email $(\mathrm{n}=367,64 \%)$. Almost three-quarters of the patients $(n=415,72 \%)$ were at average risk for breast cancer (see Table 1) (results have not been published previously).

\section{Patient satisfaction with BreastCARE}

More than three-quarters of women said completing the risk assessment was "very easy" to complete $(n=470$, $84 \%$ ) and that the survey questions were "very easy to understand" ( $\mathrm{n}=459,82 \%)$. A significantly greater proportion of white and African American women reported satisfaction compared to the other groups $(\mathrm{P}=0.001)$ (see Table 2).

The majority of participants $(n=321,61 \%)$ reported liking the breast cancer risk report "a lot", with a significantly greater proportion of Latina and African Americans providing this response compared to white and Asian American women. While more than three-quarters of women ( $n=436,80 \%)$ said the report was "very easy to understand", a greater proportion of white women did so compared to other groups $(\mathrm{P}=0.001)$.

In multivariable logistic regression analysis adjusted for clinic site, computer use, age, language, education, and objective breast cancer risk, Asian American women were less likely to report that the risk assessment questions were "very easy to understand" (OR $=0.31,95 \%$ CI: 0.15-0.66). Compared with those with a lower educational attainment, women with some college or more than a college education were more likely to report that the survey questions were very easy to understand (OR $=3.01,95 \% \mathrm{CI}: 1.52-5.95$ and $\mathrm{OR}=1.33$, 95\% CI: 1.33-3.59, respectively) (see Table 3).

Latina women, compared to non-Latina white women, were significantly more likely to report liking the breast cancer risk report "a lot" (OR =2.04, 95\% CI: 1.05-3.96), as were older women (OR =2.06, 95\% CI: 1.16-3.69).

Women at high risk were less likely to report liking the personalized risk report a lot compared to women at lower risk $(\mathrm{OR}=0.61,95 \% \mathrm{CI}: 0.40-0.92)$.

Women who indicated that they use a computer sometimes or always were more likely to report that they found the report "very easy to understand" (OR $=2.28,95 \%$ CI: $1.30-042)$.

\section{Patient preferences for receiving BreastCARE assessment and report}

A majority of patients $(n=339,59 \%)$ preferred completing the risk assessment tool on a computer. Half of the women $(\mathrm{n}=280,50 \%)$ preferred completing the risk assessment in the waiting room before seeing the doctor, with a significantly larger proportion of Latinas reporting so as compared to white women $(\mathrm{P}=0.001)$. More than two-thirds of women (72\%) preferred to receive the risk report before rather than after a doctor's visit. A greater proportion of white women expressed this preference compared to other groups $(\mathrm{P}=0.003)$ (see Table 2$)$. 
Table 1 Demographic and health information of patient sample (24)

\begin{tabular}{|c|c|c|c|c|c|c|}
\hline Indicators & $\begin{array}{l}\text { White }(n=202) \\
n(\%)^{\star}\end{array}$ & $\begin{array}{l}\text { Latina/Hispanic } \\
(\mathrm{n}=141), \mathrm{n}(\%)^{*}\end{array}$ & $\begin{array}{l}\text { Black/African American } \\
\qquad(n=125), n(\%)^{\star}\end{array}$ & $\begin{array}{l}\text { Asian American } \\
(\mathrm{n}=105), \mathrm{n}(\%)^{\star}\end{array}$ & $\begin{array}{l}\text { Total }(n=573) \\
n(\%)^{\star}\end{array}$ & $P$ value \\
\hline \multicolumn{7}{|l|}{ Demographic characteristics } \\
\hline$<50$ years & $58(28.7)$ & $52(36.9)$ & $32(25.6)$ & $28(26.6)$ & $170(29.7)$ & \\
\hline Marital status & & & & & & $<0.0001$ \\
\hline Married/living with a partner & $113(56.5)$ & $65(46.1)$ & $23(18.4)$ & $57(54.8)$ & $258(45.3)$ & \\
\hline Not married or living with partner & $87(43.5)$ & $76(53.9)$ & $102(81.6)$ & $47(45.2)$ & $312(54.7)$ & \\
\hline College degree or higher & $144(72.0)$ & $19(13.8)$ & $19(15.2)$ & $51(48.6)$ & $223(40.0)$ & \\
\hline Language of interview & & & & & & $<0.0001$ \\
\hline English & $202(100.0)$ & $67(47.5)$ & $125(100.0)$ & $69(65.7)$ & $463(87.3)$ & \\
\hline Spanish or Chinese & 0 & $74(52.5)$ & 0 & $36(34.3)$ & $110(12.7)$ & \\
\hline \multicolumn{7}{|l|}{ Access to electronic information } \\
\hline $\begin{array}{l}\text { Somewhat/very comfortable with } \\
\text { computers }\end{array}$ & $183(91.0)$ & $66(47.5)$ & $72(57.6)$ & $62(59.6)$ & $383(67.7)$ & $<0.0001$ \\
\hline UCSF & $193(95.5)$ & $54(38.3)$ & $88(70.4)$ & $73(69.5)$ & 408 (71.2) & $<0.0001$ \\
\hline $\begin{array}{l}\text { Zuckerberg San Francisco } \\
\text { General }\end{array}$ & $9(4.5)$ & $87(61.7)$ & $37(29.6)$ & $32(30.4)$ & $165(28.8)$ & \\
\hline \multicolumn{7}{|l|}{ Assessment of risk for breast cancer } \\
\hline \multicolumn{7}{|l|}{ Risk category for breast cancer } \\
\hline Average risk & $120(59.4)$ & $116(82.3)$ & $100(80.0)$ & $79(75.2)$ & $415(72.4)$ & $<0.0001$ \\
\hline High-risk, Gail/BCSC & $56(27.7)$ & $9(6.4)$ & $17(13.6)$ & $19(18.1)$ & $101(17.6)$ & $<0.0001$ \\
\hline High-risk, RST $\geq 2$ & $26(12.9)$ & $16(11.3)$ & $8(6.4)$ & $7(6.7)$ & $57(10.0)$ & $<0.0001$ \\
\hline
\end{tabular}

*, Percentages based on non-missing values. UCSF, University of California, San Francisco; BCSC, Breast Cancer Surveillance Consortium; RST, Referral Screening Tool for breast/ovarian cancer genetics. 
Table 2 Patient satisfaction with intervention and preferences for receipt by race/ethnicity

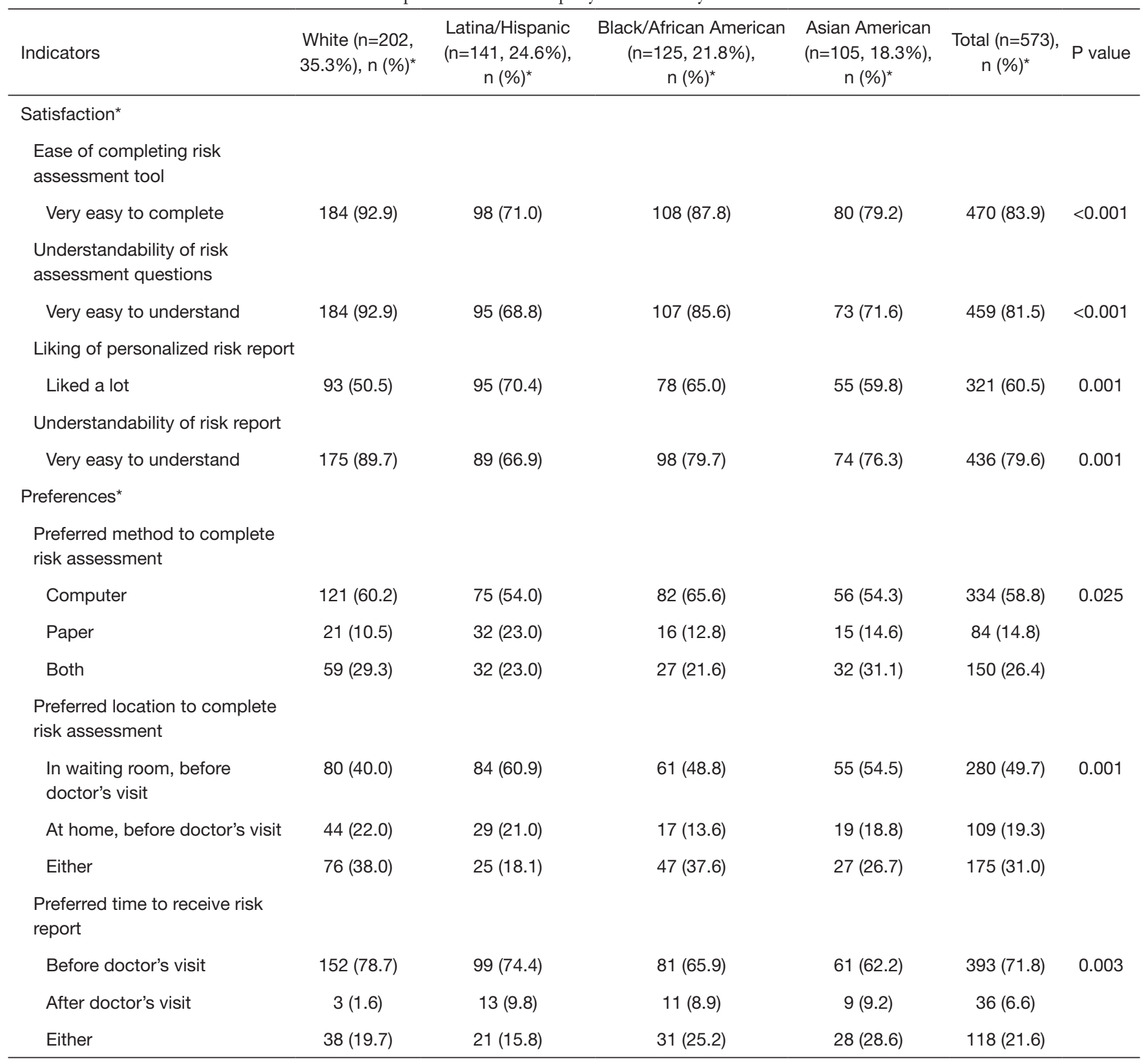

*, Percentages based on non-missing values.

\section{Physician sample}

Surveys were mailed to 223 physicians whose patients had received the intervention. Of these, we received 140 completed surveys (62.8\%). There were 16 invalid addresses and 34 participants who were no longer at UCSF (residents who graduated from the program).

\section{Physician characteristics}

The majority of physicians $(\mathrm{n}=85,60.7 \%)$ were internal medicine residents and fellows, while $39 \%(n=55)$ were attending physicians and NPs. More than half of the physician respondents $(n=83,62 \%)$ were women. Sixtyone percent of physicians identified as white $(\mathrm{n}=83)$. Nearly 
Table 3 Logistic regression of patient satisfaction indicators

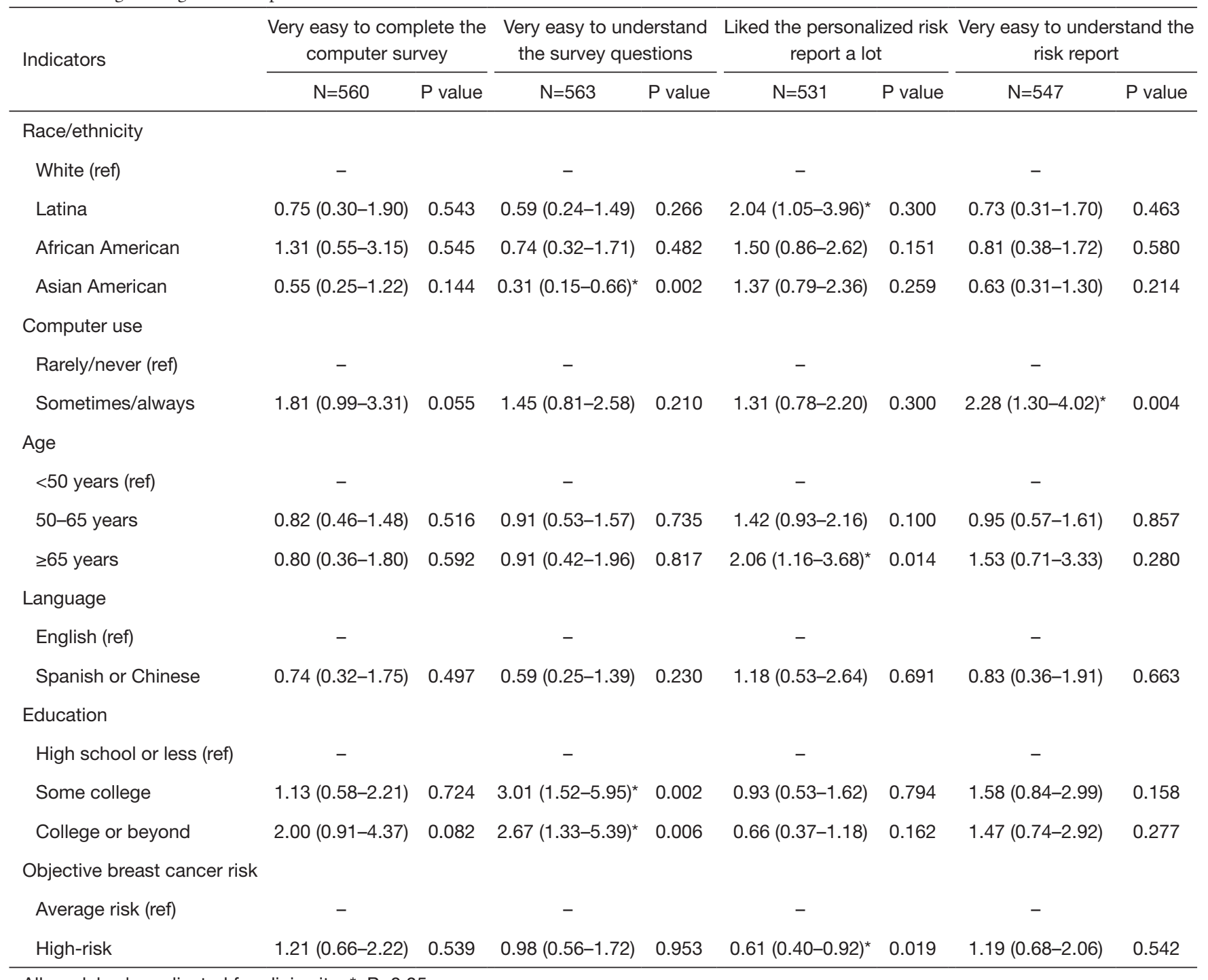

All models also adjusted for clinic site. * $\mathrm{P}<0.05$.

three-quarters $(\mathrm{n}=99,71 \%)$ reported that more than half of their female patients were between 40 and 74 years of age. Attending physicians/NPs had, on average, 9.2 years of experience (SD: 8.2) since completing their training. Overall, the majority of physicians' time was spent in direct clinical care (see Table 4).

\section{Physician acceptability}

Slightly more than half of the respondents evaluated the reports $(\mathrm{n}=79,56 \%)$. The majority of physicians, felt that the reports helped inform their patients about personal breast cancer risk ( $\mathrm{n}=68,86 \%)$, prompted them to discuss breast cancer risk with patients ( $\mathrm{n}=66,84 \%)$, helped them communicate with patients about breast cancer risk $(\mathrm{n}=63$, $84 \%$ ), and supported them in their efforts to provide preventive care for their patients ( $\mathrm{n}=59,78 \%)$.

Among the less positive perceptions of the reports, physicians believed their patients found them confusing $(\mathrm{n}=38,61 \%)$, made their patients more anxious $(\mathrm{n}=24$, $36 \%)$, kept physicians from addressing other important topics during the visit $(n=29,25 \%)$, added substantially to the length of the visit $(n=19,24 \%)$, and interfered with the visit $(\mathrm{n}=13,17 \%)$.

A significantly greater proportion of residents compared to attending physicians/NPs indicated that the reports 
Table 4 Demographic information-physician samples

\begin{tabular}{|c|c|c|c|c|}
\hline Indicators & Residents* $(n=85)$ & Attending physicians/NPs $(n=55)$ & Total sample* $(n=140)$ & $P$ value \\
\hline Men & $34(41.0)$ & $18(34.6)$ & $52(38.5)$ & 0.461 \\
\hline Women & $49(59.0)$ & $34(65.4)$ & $83(61.5)$ & \\
\hline \multicolumn{5}{|l|}{ Racial/ethnic background* } \\
\hline Latin/Hispanic & $7(8.4)$ & $3(5.7)$ & $10(7.4)$ & \\
\hline Black/African American & $3(3.6)$ & $4(7.5)$ & $7(5.1)$ & \\
\hline Asian American & $17(20.5)$ & $16(30.2)$ & $33(24.3)$ & \\
\hline Other & $3(3.6)$ & $1(1.9)$ & $4(2.9)$ & \\
\hline $26-50 \%$ & $20(24.1)$ & $8(16.0)$ & $28(21.1)$ & \\
\hline More than $50 \%$ & $58(69.9)$ & $41(82.0)$ & $99(74.4)$ & \\
\hline Physician practice characteristics* ${ }^{*}$ & & & Mean (SD) & \\
\hline Years practicing since residency & NA & $9.2(8.2)$ & $9.2(8.2)$ & NA \\
\hline Direct patient care: average hour per week & $74.8(26.4)$ & $38.6(29.0)$ & $60.3(32.5)$ & 0.000 \\
\hline
\end{tabular}

*, Percentages based on non-missing values. NP, nurse practitioner.

helped them to communicate with their patients about their breast cancer risk $(\mathrm{n}=38,97 \%$ vs. $\mathrm{n}=25,69 \%, \mathrm{P}=0.004)$. There were no other differences in acceptability by physician status (resident $v s$. attending) (see Table 5).

Of those who rated the report $(n=67)$, most rated both the physician report and patient report as "good" or "very good" ( $\mathrm{n}=54,81 \%$ and $82 \%$, respectively). No differences were found in ratings between residents and attending physicians/NPs. With respect to implementing the intervention at their site, only $7(9 \%)$ indicated that they would not implement any of the components, while 74 (91\%) indicated that they would implement at least one of the BreastCARE components.

\section{Suggestions for implementation}

Most physician respondents $(n=93,73 \%)$ felt that the primary care or gynecology practice was the best place to give patients information about breast cancer risk, although this differed significantly according to resident/attending status $(\mathrm{n}=52,65 \%$ of residents $v s . \mathrm{n}=41,85 \%$ of attending physicians/NPs; $\mathrm{P}=0.04)$. Just under half $(\mathrm{n}=62,48 \%)$ felt that the primary care physician or gynecologist would be the best practitioner to deliver the information (see Table 5).

\section{Discussion}

This study assessed the acceptability of a tablet-based breast cancer risk assessment and educational intervention in the primary care setting for both patients and physicians and identified suggestions for implementation. The intervention was implemented at two sites among patients of diverse levels of education, computer literacy, and race/ethnicity. Our results indicate that the intervention was largely acceptable and feasible across this diverse population of patients and their physicians, although some areas for improvement were identified.

We first assessed whether a tablet-based risk assessment was a feasible and acceptable way of eliciting information concerning risk among patients. The multivariate analysis found that all racial/ethnic groups responded similarly to the different satisfaction components, with a few exceptions. No racial/ethnic differences were identified in ease of completing the survey. All components of the intervention 
Table 5 Physician acceptability of BreastCARE reports and barriers to risk discussions by status (resident $v s$. attending)

\begin{tabular}{|c|c|c|c|c|}
\hline Indicators & Residents & Attending physicians/NPs & Total sample & $P$ value \\
\hline \multicolumn{5}{|l|}{ Physician acceptability } \\
\hline Perceptions of BreastCARE risk reports (agree strongly/somewhat) ${ }^{c}$ & $(n=42)$ & $(n=37)$ & $(n=79)$ & \\
\hline Helps to inform patients about their personal breast cancer risk & $38(90.5)$ & $30(81.1)$ & $68(86.1)$ & 0.229 \\
\hline Prompts discussion of breast cancer risk with my patients & $37(88.1)$ & $29(78.4)$ & $66(83.5)$ & 0.369 \\
\hline Supports efforts to do preventive care for my patients & $34(87.2)$ & $25(67.6)$ & $59(77.6)$ & 0.121 \\
\hline Patients find the reports confusing & $18(58.1)$ & $20(64.5)$ & $38(61.3))$ & 0.575 \\
\hline Makes patients anxious & $11(30.6)$ & $13(41.9)$ & $24(35.8)$ & 0.357 \\
\hline Adds time & $8(20.0)$ & $11(29.7)$ & $19(24.7)$ & 0.434 \\
\hline \multicolumn{5}{|l|}{ Rating of the physician and patient reports } \\
\hline Rating of the physician report ${ }^{a}$ & $(n=34)$ & $(n=33)$ & $(n=67)$ & \\
\hline Very good/good & $30(88.2)$ & $24(72.7)$ & $54(80.6)$ & 0.109 \\
\hline Rating of patient report ${ }^{\mathrm{b}}$ & $(n=36)$ & $(n=30)$ & $(n=66)$ & \\
\hline Very good/good & $32(94.1)$ & $22(73.3)$ & $54(81.8)$ & 0.187 \\
\hline Implementation at the site (among those who rated both reports) & $(n=44)$ & $(\mathrm{N}=37)$ & $(n=81)$ & \\
\hline None & $5(11.4)$ & $3(5.4)$ & $7(8.6)$ & \\
\hline At least one & 39(88.6) & 35 (94.6) & $74(91.4)$ & 0.342 \\
\hline Best person to give patients breast cancer risk information & $(n=80)$ & $(n=49)$ & $(n=129)$ & \\
\hline Their primary care provider or gynecologist & $39(48.8)$ & $23(46.9)$ & $62(48.1)$ & 0.084 \\
\hline Trained staff within the primary care or gynecology clinics & $21(26.3)$ & $22(44.9)$ & $43(33.3)$ & \\
\hline Trained staff within the mammography clinic & $17(21.3)$ & $4(8.2)$ & $21(16.3)$ & \\
\hline Other trained professional by phone & $2(2.5)$ & 0 & $2(1.6)$ & \\
\hline Radiologist at mammography clinic & $1(1.3)$ & 0 & $1(0.8)$ & \\
\hline
\end{tabular}

${ }^{a}$, among physicians who recalled receiving physician report. ${ }^{b}$, among physicians who recalled receiving patient report. ${ }^{c}$, among physicians who recalled receiving either physician OR patient report. BreastCARE, Breast Cancer Risk Education Intervention; NP, nurse practitioner. 
were designed to balance the needs of those who are computer literate and comfortable with a touch screen with those who have limited computer skills, which may explain why satisfaction was largely similar across racial/ethnic groups in adjusted analyses, regardless of computer literacy. Other studies indicate that, with a little help, vulnerable populations can overcome these difficulties (27). However, we did find that Asian American women indicated less understanding of the assessment tool questions, even after adjustment for educational level and access to electronic information. The reason for this remains unclear. The other groups, including Latina and African American women, who also reported lower educational attainment and less access to electronic information, reported similar levels of satisfaction to whites after multivariate adjustment.

Overall, patients from different racial/ethnic groups were also similarly satisfied with the risk report. The report was particularly well-liked by Latina women and older participants. In contrast, those at high risk for breast cancer had a more negative view of the report. It is possible that because the report was designed for low-literacy patients, providing only the necessary information to promote discussion between physicians and patients, those who were at higher risk or more educated may have been less satisfied with the depth of information provided.

Overall, the physicians who were exposed to both the patient and physician reports also reported a positive view of the intervention, and indicated that they were good tools to help inform patients and promote discussion. The resident physicians expressed greater support for the reports as a means to help communicate with patients about breast cancer risk, perhaps reflecting this group's need for additional information and support. The percentage that indicated potential barriers to implementation such as adding time to the visit or interfering with the patient visit was relatively small, representing approximately one-quarter of the physicians. These concerns are not unique to this intervention and it is unclear how much of an impediment this would be to integrating the intervention into clinical practice. The large percentage of physicians expressing support for and acceptability of the intervention suggests that it would be accepted.

Both women and physicians were asked about the best method and location to complete the risk assessment. Almost all women preferred to complete the task using a computer tablet, supporting the idea that mHealth applications are relevant for diverse patient groups. Most women also preferred that all elements of the intervention be conducted at the clinic site prior to the visit rather than at home or in other settings. This was supported by physicians' suggestions that the intervention be delivered in the context of primary care, preferably by a physician. A greater proportion of residents versus attending physicians/ NPs considered other environments acceptable for providing the information.

While most mHealth interventions are geared for home implementation, results from this study suggest that patients may prefer to complete medical assessments at the practice and just prior to seeing their health care provider, particularly if the task may generate some distress. The rationale may be similar for physicians who are concerned with the stress generated by a risk assessment tool. As clinic visits are increasingly shifting to a virtual platform, it will be important to reassess whether the intervention could be implemented at home.

While a patient may experience some degree of discomfort with receipt of risk results, the delivery and the discussion of the results in the context of the primary care setting may diminish this discomfort. A minority of physicians felt that the reports increased patient anxiety about breast cancer or believed patients would find the reports too confusing or difficult to understand. However, in a separate analysis exploring the impact of the BreastCARE intervention among patients, we actually observed an increase in knowledge about breast cancer risk factors and no increase in concern about breast cancer among women who were assigned to the intervention group compared to women who did not receive the risk reports (25).

As in prior studies, physicians cited insufficient time and training as barriers to delivering and discussing breast cancer risk information with patients (28). Insufficient time is linked to the complexity of the visit, particularly in primary care practice where patients and physicians have to balance many competing demands during a single visit (28). In these cases, preventive care may be set aside for future visits or not discussed at all $(29,30)$. Given that in today's healthcare system primary care visits are typically limited to about 20 minutes and much needs to be accomplished in a single visit, it is not surprising that about a quarter of physicians felt that the time required to discuss the results either drew time away from other important issues or added time to the visit. One way to address this concern would be to designate a trained provider, such as a clinical nurse or a health coach embedded in the practice, to discuss the results with patients, providing patient education without significantly detracting from a physician's clinical schedule. 
However, there appears to be disagreement among physicians as to whether the individual discussing breast cancer risk with the patient should be a physician or another trained staff member in the practice. Some physicians may be driven by the desire to educate their patients about breast cancer risk themselves, while others would prefer to defer some of these responsibilities to other members of the team. It may also depend on whether the patient is at high risk or not, with physicians preferring to discuss risk assessment with high-risk patients themselves.

There are several limitations to our study. The study was implemented in two academic teaching practices, so the findings may not be generalizable to other settings or to areas outside of San Francisco. The evaluation of the intervention took place within a week after delivery, which may have led to a participant socially desirable responses or recall bias. A majority of the clinicians surveyed were residents, and their responses may not reflect those of physicians with more experience. Further, we administered the physician survey 1 month after patient enrollment. This may be responsible for our response rate of $62.8 \%$. This time delay may also explain why only half of the physicians recalled receiving the report, and may limit the generalizability of the findings.

\section{Conclusions}

We found that a tablet-based breast cancer risk assessment tool, coupled with an on-site report of results, is generally easy for patients to complete and is acceptable to both patients and physicians in the primary care setting. The BreastCARE intervention was able to successfully elicit complex information from patients in an efficient manner and provide physicians with useful information at the time of the visit with patients. Next steps include scaling and dissemination of the intervention with integration into electronic health record systems for seamless implementation.

\section{Acknowledgments}

Funding: This research was funded by the California Breast Cancer Research Program (150B-0158) and Susan G. Komen for the Cure (KG090504). Both trials were recorded under one NCT identifier (NCT01830933) based on recommendations from NIH ClinicalTrials.gov. Drs. CPK and LK are supported by the National Aging Institute funded Center for Aging in Diverse Communities at the University of California San Francisco (P30 AG15272).
Drs. CPK and JLT a had full access to all of the data in the study and take responsibility for the integrity of the data and the accuracy of the data analysis.

\section{Footnote}

Data Sharing Statement: Available at http://dx.doi. org/10.21037/mhealth-20-82

Conflicts of Interest: All authors have completed the ICMJE uniform disclosure form (available at http://dx.doi. org/10.21037/mhealth-20-82). Drs. JLT and JAT report grants from California Breast Cancer Research Program, grants from Susan G. Komen for the Cure, during the conduct of the study. The other authors have no conflicts of interest to declare.

Ethical Statement: The authors are accountable for all aspects of the work in ensuring that questions related to the accuracy or integrity of any part of the work are appropriately investigated and resolved. The study was conducted in accordance with the Declaration of Helsinki (as revised in 2013). The study was approved by the Institutional Review Board (IRB) of UCSF (project number 10-01288;10-00129) and informed consent was taken from all the participants.

Open Access Statement: This is an Open Access article distributed in accordance with the Creative Commons Attribution-NonCommercial-NoDerivs 4.0 International License (CC BY-NC-ND 4.0), which permits the noncommercial replication and distribution of the article with the strict proviso that no changes or edits are made and the original work is properly cited (including links to both the formal publication through the relevant DOI and the license). See: https://creativecommons.org/licenses/by-nc-nd/4.0/.

\section{References}

1. Tice JA, Cummings SR, Smith-Bindman R, et al. Using clinical factors and mammographic breast density to estimate breast cancer risk: development and validation of a new predictive model. Ann Intern Med 2008;148:337-47.

2. Ozanne EM, Klemp JR, Esserman LJ. Breast cancer risk assessment and prevention: a framework for shared decision-making consultations. Breast J 2006;12:103-13.

3. Antoniou AC, Cunningham AP, Peto J, et al. The BOADICEA model of genetic susceptibility to breast 
and ovarian cancers: updates and extensions. Br J Cancer 2008;98:1457-66.

4. Srivastava A, McKinnon W, Wood ME. Risk of breast and ovarian cancer in women with strong family histories. Oncology (Williston Park) 2001;15:889-902.

5. Berry DA, Iversen ES Jr, Gudbjartsson DF, et al. BRCAPRO validation, sensitivity of genetic testing of BRCA1/BRCA2, and prevalence of other breast cancer susceptibility genes. J Clin Oncol 2002;20:2701-12.

6. Gail MH, Brinton LA, Byar DP, et al. Projecting individualized probabilities of developing breast cancer for white females who are being examined annually. J Natl Cancer Inst 1989;81:1879-86.

7. Tice JA, Cummings SR, Ziv E, et al. Mammographic breast density and the Gail model for breast cancer risk prediction in a screening population. Breast Cancer Res Treat 2005;94:115-22.

8. Bellcross CA, Lemke AA, Pape LS, et al. Evaluation of a breast/ovarian cancer genetics referral screening tool in a mammography population. Genet Med 2009; 11:783-9.

9. Vogel VG, Costantino JP, Wickerham DL, et al. Effects of tamoxifen vs raloxifene on the risk of developing invasive breast cancer and other disease outcomes: the NSABP Study of Tamoxifen and Raloxifene (STAR) P-2 trial. JAMA 2006;295:2727-41.

10. Chlebowski RT, Col N, Winer EP, et al. American Society of Clinical Oncology technology assessment of pharmacologic interventions for breast cancer risk reduction including tamoxifen, raloxifene, and aromatase inhibition. J Clin Oncol 2002;20:3328-43.

11. Kinsinger LS, Harris R, Woolf SH, et al. Chemoprevention of breast cancer: a summary of the evidence for the U.S. Preventive Services Task Force. Ann Intern Med 2002;137:59-69.

12. Behan LA, Amir E, Casper RF. Aromatase inhibitors for prevention of breast cancer in postmenopausal women: a narrative review. Menopause 2015;22:342-50.

13. Carbine NE, Lostumbo L, Wallace J, et al. Risk-reducing mastectomy for the prevention of primary breast cancer. Cochrane Database Syst Rev 2018;4:CD002748.

14. Kaplan CP, Haas JS, Perez-Stable EJ, et al. Breast cancer risk reduction options: awareness, discussion, and use among women from four ethnic groups. Cancer Epidemiol Biomarkers Prev 2006;15:162-6.

15. Kaplan CP, Haas JS, Perez-Stable EJ, et al. Factors affecting breast cancer risk reduction practices among California physicians. Prev Med 2005;41:7-15.
16. Armstrong K, Quistberg DA, Micco E, et al. Prescription of tamoxifen for breast cancer prevention by primary care physicians. Arch Intern Med 2006;166:2260-5.

17. Turnin MC, Beddok RH, Clottes JP, et al. Telematic expert system Diabeto. New tool for diet self-monitoring for diabetic patients. Diabetes Care 1992;15:204-12.

18. Gustafson DH, Hawkins R, Pingree S, et al. Effect of computer support on younger women with breast cancer. J Gen Intern Med 2001;16:435-45.

19. Calfas KJ, Sallis JF, Zabinski MF, et al. Preliminary evaluation of a multicomponent program for nutrition and physical activity change in primary care: PACE+ for adults. Prev Med 2002;34:153-61.

20. Green MJ, Peterson SK, Baker MW, et al. Use of an educational computer program before genetic counseling for breast cancer susceptibility: effects on duration and content of counseling sessions. Genet Med 2005;7:221-9.

21. Gustafson DH, Hawkins R, Boberg E, et al. Impact of a patient-centered, computer-based health information/ support system. Am J Prev Med 1999;16:1-9.

22. Green MJ, Peterson SK, Baker MW, et al. Effect of a computer-based decision aid on knowledge, perceptions, and intentions about genetic testing for breast cancer susceptibility: a randomized controlled trial. JAMA 2004;292:442-52.

23. Sweet KM, Bradley TL, Westman JA. Identification and referral of families at high risk for cancer susceptibility. J Clin Oncol 2002;20:528-37.

24. Kaplan CP, Livaudais-Toman J, Tice JA, et al. A randomized, controlled trial to increase discussion of breast cancer in primary care. Cancer Epidemiol Biomarkers Prev 2014;23:1245-53.

25. Livaudais-Toman J, Karliner LS, Tice JA, et al. Impact of a primary care based intervention on breast cancer knowledge, risk perception and concern: a randomized, controlled trial. Breast 2015;24:758-66.

26. Research J. Jambeyang Research Innovation for Healthcare. 2020. [cited 2020 October 25, 2020]. Available online: https://www.jambeyang.com/

27. Bravo C, O'Donoghue C, Kaplan CP, et al. Can mHealth improve risk assessment in underserved populations? Acceptability of a breast health questionnaire app in ethnically diverse, older, low-income women. J Health Dispar Res Pract 2014;7:4-19.

28. Yarnall KS, Pollak KI, Ostbye T, et al. Primary care: is there enough time for prevention? Am J Public Health 2003;93:635-41. 
29. Braddock CH, 3rd, Snyder L. The doctor will see you shortly. The ethical significance of time for the patient-physician relationship. J Gen Intern Med 2005;20:1057-62.

doi: $10.21037 /$ mhealth-20-82

Cite this article as: Kaplan CP, Karliner L, Lee A, LivaudaisToman J, Tice JA, Ozanne E. Acceptability of an mHealth breast cancer risk-reduction intervention promoting risk assessment, education, and discussion of risk in the primary care setting. mHealth 2021;7:54.
30. Dugdale DC, Epstein R, Pantilat SZ. Time and the patient-physician relationship. J Gen Intern Med 1999;14 Suppl 1:S34-40. 